\title{
The incidence of soft tissue sarcoma in Dakshina Kannada: study in a District Government Hospital
}

\author{
Ashish Gupta · Harish K. Rao · Soumya Gupta
}

Received: 10 October 2008 / Accepted: 24 December 2008

(C) Association of Surgeons of India 2009

\begin{abstract}
Background We present a retrospective study depicting the incidence and outcome of soft tissue sarcomas (STSs) in patients admitted in a District Government Hospital situated in coastal belt of Southern India for a period of four and a half years. The hospital is a district referral centre catering to rural and urban poor population of $1,900,000$ people.
\end{abstract}

Methods Histologically proven soft tissue STS patients admitted in Department of General Surgery in District Government Wenlock Hospital, Mangalore, from January 2002 to July 2007 were included in the study. The incidence, age distribution, gender distribution, histological subtypes, site of tumour, and clinical outcome were the parameters studied. The above parameters were then compared with Memorial Sloan and Kettering Cancer Centre (MSKCC) study.

Results Fifty-one cases of STS were reported out of 7674 $(0.65 \%)$ patients with cancer in the said period. Ninety percent belonged to adolescents and adult age group. Liposarcoma $(18 \%)$ is the most common subtype followed

A. Gupta ${ }^{1} \cdot$ H. K. Rao ${ }^{2} \cdot$ S. Gupta

${ }^{1}$ Department of General Surgery,

Department of Plastic Surgery,

D.M.C. and Hospital,

Ludhiana, India

${ }^{2}$ Department of General Surgery,

Kasturba Medical College,

Mangalore, India

A. Gupta $(\bowtie)$
E-mail: docashish2001@gmail.com by leiomyosarcoma, Ewings' sarcoma. $66.6 \%$ originated in the extremities and rest being intra-abdominal and retroperitoneal. The age of presentation was a decade less than MSKCC study. The alarming yet expected fact was 35\% of patients came with delayed presentation of the disease and refusal for surgery. Thirty-nine percent of patients were treated surgically.

Conclusion Majority of patients presenting to our institution in advanced stage of the disease, indicating ignorance, fear and reluctance for surgery; as well as economic constraints, that delay early detection and initiation of proper treatment. The incidence appears to be increasing, targeting the younger population. There is a definite need to incorporate drug trials in rural set up so that patients can be benefited.

Keywords Soft tissue sarcoma $\cdot$ Incidence $\cdot$ Dakshina Kannada $\cdot$ District Government Wenlock Hospital

\section{Introduction}

Soft tissues sarcomas (STS) are heterogeneous group of tumours with more than 50 histological subtypes. According to the western literature they account for approximately $1 \%$ of all adult cancers [1]. Although by total number STS crests in fifth decade of life, by percent of cancers for a given age, they peak in the childhood and adolescence, where they account for approximately $8 \%$ of all cancers. These tumours may develop at any anatomical site, approximately $50 \%$ occur in extremities followed in order of frequency by the abdominal cavity/retroperitoneum, trunk/thoracic region and head and neck. 
Clinical examination, trucut biopsy, CT and/or MRI is required for diagnosis as well as staging of the disease. Current staging systems (AJCC) focus on histological grade of tumour, size of the primary tumour and presence or absence of metastasis [2-4]. Histological grade is a major prognostic determinant and is based on cellularity, differentiation, polymorphisms, necrosis and number of mitosis. The potential cure of this disease is inversely proportional to the volume and spread of the disease. Surgery is the mainstay of treatment in most of STS. Patients presenting with distant metastasis have poor prognosis in spite of chemotherapy and/or surgery. Reporting and data collection of incidence, presentation and clinical outcome of patients with STS in rural and urban poor population of India is inadequate as National Cancer Registry System is still in infancy with poor inflow of data from the teeming masses. We are presenting the incidence, predominant histological types and stage of presentation of patients with STS coming to our District Government Hospital from January 2002 to July 2007 catering to a diverse population of 1,900,000 people of Dakshina Kannada (Pre-independence; Southern Karnataka, Northern Kerala), mainly the densely populated coastal belt on the south-western frontier overlooking the Arabian sea with versatile geography of the Western Ghats.

\section{Materials and methods}

A retrospective study conducted in Government District Referral Hospital of South Karnataka covering an area of $4650 \mathrm{sq} \mathrm{km}$ of south-western coastal India. Patients included in the study were non-skeletal STS (histologically proven), presenting or referred to our institution to the Department of General Surgery. Patients diagnosed with bone tumours were not included in the study. Patients treated on OPD basis or patients refusing admission were excluded from the study. More than $90 \%$ of case were of low socioeconomic status [annual income less than Rs.12,000 (\$300)] residing in rural and tribal areas and the urban poor. Patients were investigated in the form of routine blood investigations, chest X-ray, ultrasound abdomen, incision biopsy and $\mathrm{CT}$ scan (if patient was affordable). The disease was staged and graded and accordingly treated. The treatment provided was free of cost which included hospital stay, surgery, chemotherapy (if indicated) and rehabilitation.

Patients who refused admission or those treated on op basis were not included in the study. Other criteria for exclusion were the patients with bone tumours. The data obtained was then compared with Memorial Sloan and Kettering Cancer Centre (MSKCC) study. This study was conducted from 1982 to 2002 and included 5069 patients with STS. Considering the shorter duration of study i.e. 5 years; the smaller number of cases in our study; and the fact that our institute is not a dedicated Cancer Research Institute; the comparison thus cited is unevenly balanced in favour of the latter, but it is our endeavour to present the incidence of this often mismanaged disease entity presenting to the Department of Surgery in a District Government Hospital.

In spite of the best efforts; health education by village health guides, primary health centres and community health centres and the various health education programmes, propagated by the Government and various Non-Government Organizations, the level of ignorance, fear of deforming surgery and loss of productivity were the leading factors for delayed presentation of the advanced disease and refusal for treatment in a set of patients. Ironically the geographical area covered by our centre has the highest literacy rate in the country.

\section{Results}

From January 2002 to July 2007 there were 87,648 admissions in District Government Wenlock Hospital of the total patients admitted, $7674(8.75 \%)$ were cases of malignancy of different systems of body. Soft tissue sarcoma accounted for $0.65 \%$ (51 patients) of the various malignancies admitted in all age groups, during the said interval (Table 1).

STS appears to the more common among adults accounting for $49 \%$ of cases (age group 31-60) (Table 2). Our statistics indicated that adolescents and adults are more affected in the population of Southern Karnataka and Northern Kerala (Table 3). The peak age of presentation was found to be fourth to fifth decade. Ninety percent of liposarcoma and $100 \%$ of leiomyosarcoma belonged to age group 31-60 years. In MSKCC study the peak age being fifth to sixth decade with $52 \%$ of liposarcoma and $57 \%$ of leiomyosarcoma in age group 31-60 years.

It was found that there was a male preponderance amongst the patients studied. The male to female ratio was 1.36:1 with males' patients numbering 29 and 22 female patients. The gender distribution according to the histopathological sub types are described in Fig. 1. There was marked male predominance in case of liposarcoma (8/9 patients) where as rest others subtypes had almost equal gender distribution.

The histological presentation is different from world statistics available in the in incidence of the most prevalent STS. In our study liposarcoma (17.5\%) followed by leiomyosarcoma (15.7\%), Ewings' sarcoma (13.75\%), then rhabdomyosarcoma. Nerve sheath tumour, malignant fibrous histiocytoma (MFH) and synovial cell sarcoma (9.8\%).

According to MSKCC, the most commonly found sarcomas were liposarcoma (18\%), MFH (18\%) and leiomyosarcoma $(17 \%)$. Malignant peripheral nerve tumours account for $3 \%$, synovial cell $7 \%$. 
The occurrence of STS was predominantly in the extremities with 34 patients $(66.6 \%)$ and intra-abdominal (5 patients, 9.8\%) and retroperitoneal (9 patients, $17.6 \%$ ) was in concurrence with data from MSKCC (Fig. 2).

Surgery was the primary modality of treatment in $39.2 \%$ (20 patients). Fifteen percent (8 patients) received chemotherapy following surgery. $17.6 \%$ (9 patients) of the patients left against medical advice. 17.6\% (9 patients) presented with terminal stage and received only symptomatic palliative treatment. This shows that $35 \%$ of cases presented late or were diagnosed late. This surely indicates the lack of awareness among people as well as health care workers regarding STS (Fig. 3).

\section{Discussion}

Amongst the innumerable neoplasms ailing the human body, STSs present as a clinical dilemma, a stiff challenge for the oncologist and the surgeon. The diversity of cell of origin in the body, rapid growth in any anatomical site and the aggressive tumour biology makes it difficult to assess and treat. The high recurrence rate makes long-term follow up and repeated interventions mandatory.

In the era of multimodality treatment, where the surgeon, who is the team leader is aided by pathologist, radiologist, medical and radiation oncologist in diagnosing and instituting appropriate, effective, available and affordable therapy at the earliest to achieve maximum disease-free survival. Even with advancements of understanding of genetic alterations like KIT mutations in GIST and APC/B-catenin mutation in desmoid tumours, no specific aetiological basis has been found [5-8]. Genetic syndromes, exposure to ionising radiations and lymph oedema are well established but uncommon antecedents to the development of STS; were obvious by their absence in the patients presenting to our institution.

Of the 7674 cases of malignancy presenting to our institution, 51 patients with STS constituted $0.65 \%$ of the

Table 1 Number of cases admitted between January 2002 to July 2007

\begin{tabular}{llll}
\hline Year & No. of cases of STS & Total admissions & Malignancy \\
\hline 2002 & 6 & 13,607 & 2534 \\
2003 & 11 & 14,681 & 1176 \\
2004 & 11 & 15,757 & 1272 \\
2005 & 8 & 15,282 & 1077 \\
2006 & 7 & 14,935 & 953 \\
2007 (July) & 8 & 13,386 & 662 \\
\hline Total & 51 & 87,648 & 7674
\end{tabular}

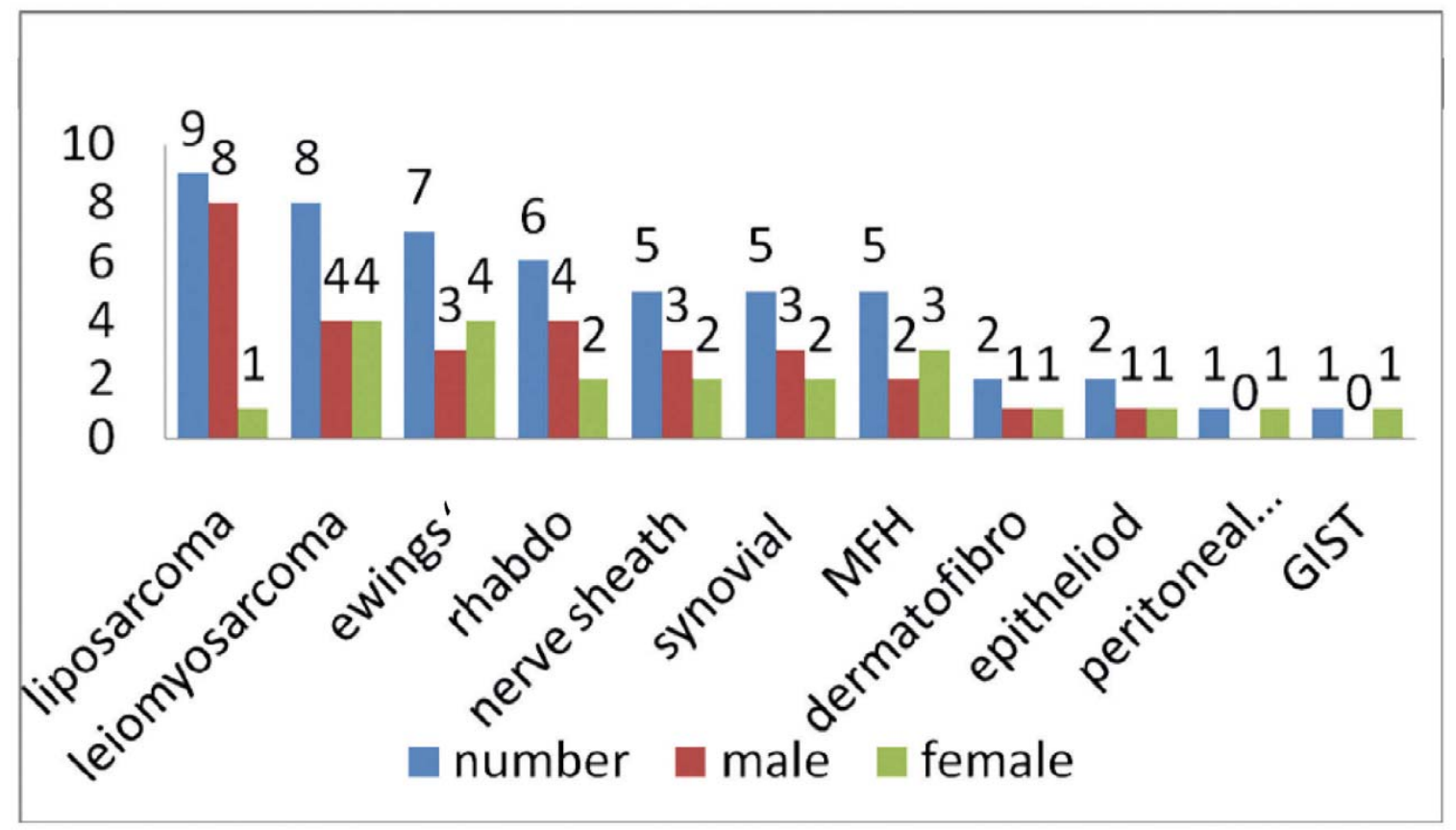

Fig. 1 Histological subtype and gender distribution 
Table 2 Age distribution

\begin{tabular}{ll}
\hline Years & No. of patients \\
\hline $1-10$ & 3 \\
$11-30$ & 20 \\
$31-60$ & 25 \\
$>61$ & 3 \\
\hline
\end{tabular}

(18\%) and leiomyosarcoma (17\%) [9]. The difference can be attributed to the small volume of cases presenting to our wards and patients not included in the study who refused admission.

Histopathology is anatomical site dependent with extremity STS most common (liposarcoma/MFH); retroperitoneal being liposarcoma and leiomyosarcoma; visceral,

Table 3 Histological type vs age of presentation

\begin{tabular}{|c|c|c|c|c|}
\hline Histological type & $1-10$ years & $11-30$ years & $31-60$ years & $>61$ years \\
\hline Liposarcoma & & & 8 & 1 \\
\hline Leiomyosarcoma & & & 8 & \\
\hline Ewings' sarcoma & & 7 & & \\
\hline Rhabdomyosarcoma & 3 & 1 & 2 & \\
\hline Nerve sheath tumor & & 2 & 2 & 1 \\
\hline Synovial cell sarcoma & & 4 & 1 & \\
\hline MFH & & 4 & 1 & \\
\hline Dermatofibrosarcoma & & 1 & & 1 \\
\hline Epithelioid sarcoma & & 1 & 1 & \\
\hline Peritoneal mesothelioma & & & 1 & \\
\hline GIST & & & 1 & \\
\hline Total & 3 & 20 & 25 & 3 \\
\hline
\end{tabular}

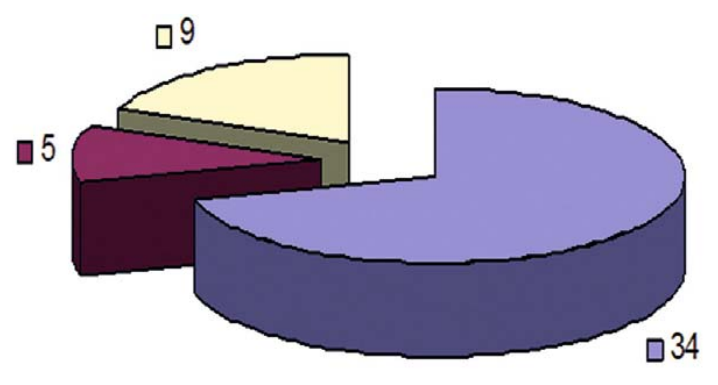

口EXTREMITIES \INTRA-ABDOMINAL QRETROPERITONEAL

Fig. 2 Site distribution

admissions from January 2002 to July 2007 as compared to $1 \%$ of adult human cancers, making it amongst the rare and unusual neoplasms [1]. There were about 11 different histological subtypes of each with clinical, therapeutic and prognostic features. The incidence of most prevalent STS in our series was different from the data provided by Memorial Sloan Kettering Cancer Centre. The incidence in descending order of frequency was liposarcoma (17.5\%), leiomyosarcoma $(15.7 \%)$ in our series. MSKCC, the most commonly found sarcomas were Liposarcoma (18\%), MFH most common being GIST. Our data was consistent with liposarcoma being most prevalent extremity sarcoma, in retroperitoneal space liposarcoma and leiomyosarcoma were commonest and GIST in visceral sarcomas [9].

STS occurred in all age groups but predominantly in adolescents and adults. Liposarcoma (8/9, 88.8\%) and leiomyosarcoma $(8 / 8,100 \%)$ occurred in age group 31-60 years. Ewings' sarcoma was exclusively found in age group 11-30 years. Rhabdomyosarcoma are known to occur in children but we saw a different trend. Fifty percent of cases were $<10$ yrs and rest $50 \%$ occurred in the $3 \mathrm{rd}$ and the 5 th decade of life. Synovial cell and MFH are common among $11-30$ years $(4 / 5)$.

Analysing the management instituted we concluded that surgery was the primary modality of treatment $(39.2 \% ; 20$ / 51). Fifteen percent received chemotherapy following surgery. $17.6 \%$ left against medical advice following diagnosis whereas $17.6 \%$ presented terminal stage and received only palliative therapy. We infer that these patients either presented late or were diagnosed late. This signifies the lack of awareness among the people and health care workers about cancers. It is also disturbing to know that although literate, patients do not understand the disease process and the need to come early in case of illness. Limited availability of diagnostic aids and its high cost are other reasons for delay in diagnosis.

At rural level the lack of specialists and limited therapeutic options are further deterrent to early diagnosis and 


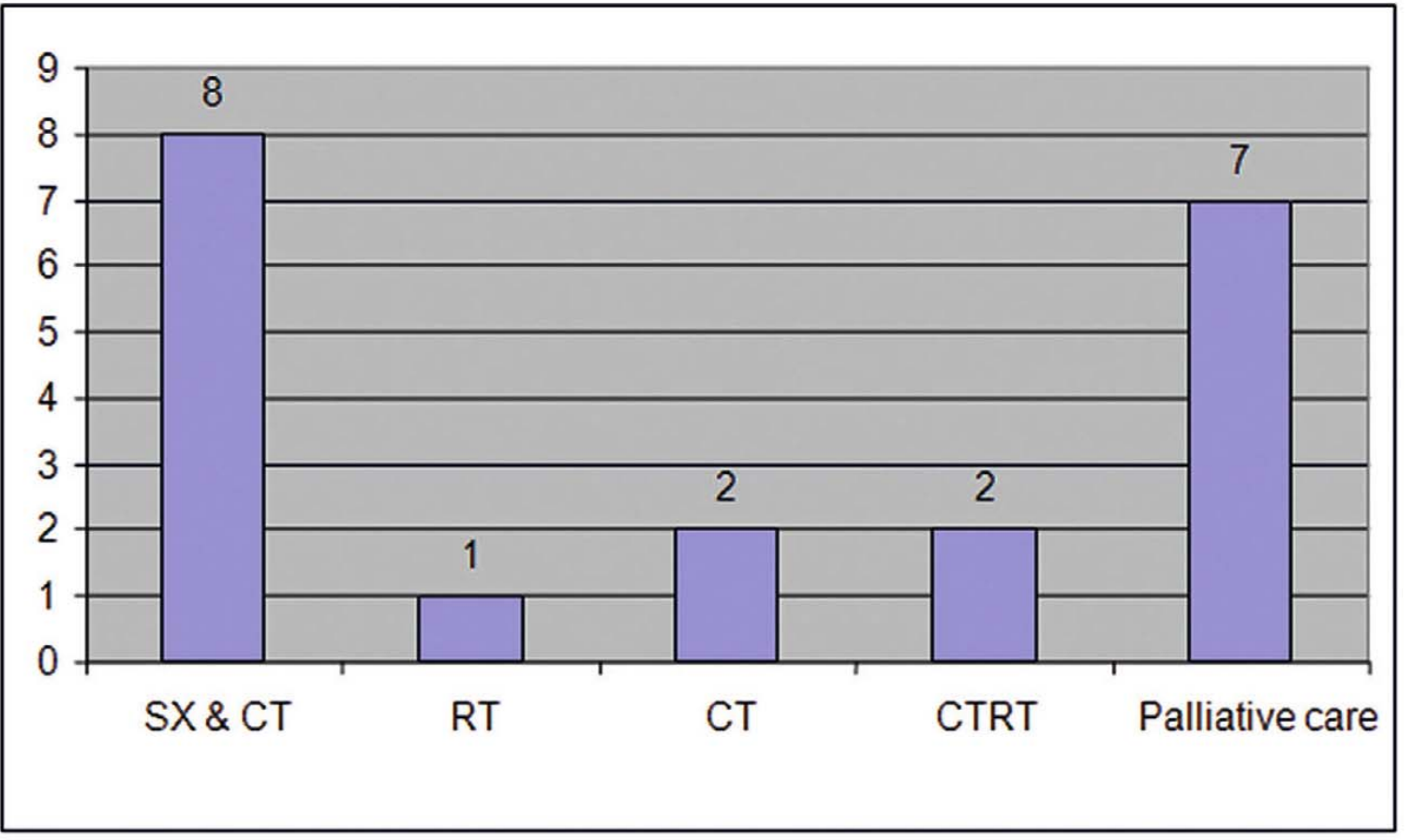

Fig. 3 Treatment provided. SX, surgery; CT, Chemotherapy; RT, Radiotherapy; CTRT, Chemotherapy and radiotherapy

institution of appropriate therapy to the patient. Another problem faced is the poor compliance to chemotherapy and/or radiotherapy and many of the rural patients are lost to follow up.

This study indicates the involvement of younger patients with STS which is an alarming and disturbing fact. The need of the hour is early detection and multidisciplinary team approach towards its treatment. It is important to set up specialist centres at rural level; most importantly, the incorporation of drug trials in government referral centres where majority of these patients present, so that they can be benefited by newer modalities of treatment at minimal cost to their families.

Conflict of interest The authors do not have any disclosable interest

\section{References}

1. Data from Surveillance, Epidemiology and End Results (SEER) Programme: SEER 9 Regs Public Use, Nov 2001 Sub [1973-1999]. Available at www.seer.cancer.gov

2. Gaynor JJ, Tan CC, Casper ES, et al. (1992) Refinement of cilinico pathological staging for localized soft tissue sar- coma of the extremity: A study of 423 adults. J Clin Oncol 10:1317

3. Fleming I, Cooper J, Henson D, et al. (1997) AJCC Cancer Staging Manual, 5th Ed. Philadelphia, Lippincott-Raven

4. Brennan MF (1999) Staging of soft tissue sarcomas. Ann Surg Oncol 6:8

5. Fletcher JA (1997) Cytogenetics of soft tissue tumours. Cancer Tret Res 91:9

6. Fletcher CD (1997) Soft tissue tumours: The impact of cyrtogenetics and molecular genetics. Verh Dtsch Ges Pathol $81: 381$

7. Sreekantaiah C, Ladanyi M, Rodriguez E, Chaganti RS (1994) Chromosomal aberrations in soft tissue tumours: Relevance to diagnosis, classification, and molecular mechanisms. Am J Pathol 144:1121

8. Meis-Kindblom JM, Sjogren H, Kindblom LG, et al. (2001) Cytogenetic and molecular genetic analysis of liposarcoma and its soft tissue simulators: Recognition of new variants and differential diagnosis. Virchows Arch 439:141

9. Beauchamp, Evers, Mattox: Sabiston Textbook of Surgery. 17th Ed. Elseveir vol. 1:805, 2007

10. Munk PL, Vellet AD, Bramwell V, et al. (1993) Soft tissue sarcoma: a plea for proper management. Can J surg 36(2): $178-180$

11. Jermal A, Tiwari RC, Murray T et al. (2004) Cancer statistics. CA Camcae J Clin 54(1):8-29 